\title{
ON THE MINIMALITY OF EXTRA CRITICAL POINTS OF GREEN FUNCTIONS ON FLAT TORI
}

\author{
CHANG-SHOU LIN AND CHIN-LUNG WANG
}

ABSTRACT. This is a sequel to $[8,2]$ to study the geometry of flat tori. In [8], we showed that the solvability of the mean field equation (MFE)

$$
\triangle u+e^{u}=\rho \delta_{0}
$$

on a flat torus $E_{\tau}$ with $\rho=8 \pi$ is equivalent to the existence of extra pair of critical points $\pm p$ of the Green function $G$. And such a pair, if exists, is unique. It was also announced there that $G$ actually attains its minimum at $\pm p$. Here our first main result is to confirm this statement by way of the variational form of the MFE. It implies that the solution $u$ is a minimizer of the corresponding non-linear functional $J_{8 \pi}(u)$ (c.f. (1.1)), hence settles the existence problem of minimizers posed in [12].

We also prove the uniqueness of solution to the MFE when $0<\rho<8 \pi$ and get the exact counting result of the number of solutions in terms of the number of critical points of $G$ when $\rho$ is close to $8 \pi$. This allows us to analyze the bifurcation structure of the MFE when $\rho$ crosses $8 \pi$.

\section{CONTENTS}

0 . Introduction

1. On the minimality of extra critical points 3

2. Computations for $D\left(\frac{1}{2} \omega_{j}\right) \quad 7$

3. Uniqueness of solutions 10

$\begin{array}{ll}\text { References } & 13\end{array}$

\section{INTRODUCTION}

Consider the flat torus $E=E_{\tau}=\mathbb{C} / \Lambda_{\tau}, \tau=a+b i, b>0$ and $\Lambda=\Lambda_{\tau}=$ $\mathbb{Z} \omega_{1}+\mathbb{Z} \omega_{2}$ with $\omega_{1}=1$ and $\omega_{2}=\tau$. Let $G$ be the Green function on $E$ :

$$
\left\{\begin{array}{l}
-\triangle G=\delta_{0}-\frac{1}{|E|} \quad \text { on } E, \\
\int_{E} G=0,
\end{array}\right.
$$

where $\delta_{0}$ is the Dirac measure at the lattice point $0 \in E$. We continue our study, initiated in $[8,2,6]$, on the critical points of $G$ :

$$
\nabla G(z)=0
$$


Since $G$ is an even function on $E$, all the half-periods $\frac{\omega_{k}}{2}$ are critical points of $G$. A critical point $p$ is called a non-trivial critical point of $G$ if $p$ is not one of the three half-periods. Clearly, non-trivial critical points appear in pair $\pm p$. It is natural to ask: how many pairs of non-trivial critical points might G have? This has been answered completely in our previous paper [8]:

Theorem A. For any $\tau \in \mathbb{H}$, the Green function $G(z ; \tau)$ on the flat torus $E_{\tau}$ has at most one pair of non-trivial critical points.

Thus $G$ has either 3 or 5 critical points. Following [8] we denote by $\Omega_{3}$ (resp. $\Omega_{5}$ ) the subset of the moduli $\mathcal{M}_{1}=\mathbb{H} / \operatorname{SL}(2, \mathbb{Z})$ where $G(z ; \tau)$ on the flat torus $E_{\tau}$ has exactly 3 (resp. 5) critical points. See $[8,7]$ for the actual shape of the (simply connected) domain $\Omega_{5}$.

What is the nature of those extra critical points? We answer it in the following theorem, which had been announced in $[8, \S 1$ Theorem $\mathrm{A}]$ :

Theorem 0.1. Suppose that the pair of non-trivial critical points $\{ \pm p\}$ of $G$ exists, then $\pm p$ are the minimal points of $G$.

We will present a proof of it in $\S 1$ based on the mean field equation

$$
\triangle u+e^{u}=8 \pi \delta_{0} \quad \text { on } E .
$$

In fact our proof shows that any solution to (0.3) must be a minimizer of the non-linear functional

$$
J_{8 \pi}(u)=\frac{1}{2} \int_{E}|\nabla u|^{2}-8 \pi \log \int_{E} e^{-8 \pi G+u}
$$

on $u \in H^{1}(E) \cap\left\{u \mid \int_{E} u=0\right\}$. This completely solves the existence problem on minimizers raised in [12] when the two vortex points collapse into one.

One important application of Theorem 0.1 is the following result:

Corollary 0.2. Suppose that the Green function G has non-trivial critical points, then all the three half periods are saddle points of $G$. That is, the Hessian of $G$ is non-positive: $\operatorname{det} D^{2} G\left(\frac{\omega_{k}}{2}\right) \leq 0$ for $k=1,2,3$.

Remark 0.3. Based on Corollary 0.2, a stronger result is proved in [7]. Namely $\operatorname{det} D^{2} G\left(\frac{\omega_{k}}{2}\right)<0$ for $k=1,2,3$ if $G$ satisfies the hypothesis of Corollary 0.2.

From the Weierstrass elliptic curve model $y^{2}=4 x^{3}-g_{2} x-g_{3}$ of $E_{\tau}$, we know that the half periods $E_{\tau}[2]$ are precisely the branch points of the map $x=\wp(z): E_{\tau} \rightarrow \mathbb{P}^{1}$. A quantity $D(q)$ defined at any branch point is strongly related to the geometry of $E_{\tau}$ at $q$. In $[1,6]$ it was proved that if $u_{k}$ is a bubbling sequence of solutions to (0.3) with $\rho=\rho_{k} \rightarrow 8 \pi$ (as $k \rightarrow \infty$ ), $\rho_{k} \neq 8 \pi$ for large $k$, and with $q$ the blow-up point, then $q$ must be a half period point. In fact, asymptotically

$$
\rho_{k}-8 \pi=(D(q)+o(1)) e^{-\lambda_{k}}
$$


where $\lambda_{k}=\max _{E_{\tau}} u_{k}$ and

$$
D(q):=\int_{E_{\tau}} \frac{h(z) e^{8 \pi(\tilde{G}(z, q)-\phi(q))}-h(q)}{|z-q|^{4}}-\int_{E_{\tau}^{c}} \frac{h(q)}{|z-q|^{4}} .
$$

Here $h(z)=e^{-8 \pi G(z)}, \tilde{G}(z, q)$ is the regular part of the Green function, and $\phi(q)=\tilde{G}(q, q)$. See $\S 2$ for more details. $D(q)$ plays an important role in the construction of bubbling solutions to (0.3), as well as in other non-linear PDEs, with $\rho_{k} \rightarrow 8 \pi$. The sign of $D(q)$ determines the direction where the bubbling may take place, namely $\rho_{k}<8 \pi$ or $\rho_{k}>8 \pi$. If $q$ is a not halfperiod critical point then $D(q)$ is still defined. But then $D(q)=0$ since $\rho_{k}=8 \pi$ for all $k$ (large).

In general it is difficult to compute $D(q)$ for a given torus. Nevertheless we will prove the following result in $\S 2$ :

Theorem 0.4. For any half period $q \in E_{\tau}, \tau=a+b i$, we have

$$
D(q)=-4 \pi^{2} b e^{-8 \pi G(q)} \operatorname{det} D^{2} G(q) .
$$

By Remark 0.3, we have $D(q)>0$ if $q$ is a saddle point. In particular if $\tau \in \Omega_{5}$ then $D(q)>0$ for all half-periods. For any $\tau \in \mathbb{H}, D(q) \leq 0$ if and only if $q$ is the minimal point.

Combining with a recent technique in analyzing uniqueness of blow-up solutions [11], we will be able to classify all solutions to (0.3) for $\rho$ in the range $\left(0,8 \pi+\epsilon_{0}\right)$ for some $\epsilon_{0}>0$ :

Theorem 0.5. For any torus $E_{\tau}$, there is a small number $\epsilon_{0}>0$ such that

(i) If $\tau \in \Omega_{3}$ then (0.3) has only one solution for $\rho<8 \pi$, no solution for $\rho=8 \pi$, and two solutions for $8 \pi<\rho<8 \pi+\epsilon_{0}$.

(ii) If $\tau \in \Omega_{5}$ then (0.3) has only one solution for $\rho<8 \pi$, infinitely many solutions for $\rho=8 \pi$, and four solutions for $8 \pi<\rho<8 \pi+\epsilon_{0}$.

In particular, the topological Leray-Schauder degree $d_{\rho}$, which is 2 for $\rho \in(8 \pi, 16 \pi)[3,4,5,6]$, does not reflect the actual number of solutions. The proof is presented in $\S 3$, which relies also on the theory of Lamé equations in [2] accompanied with (0.3) as well as the blow-up analysis in [4,11].

Remark 0.6. In [9] (see also [2]), we proved that (0.3) with $\rho=12 \pi$ has exactly two solutions on $E_{\tau}$ for $\tau \neq e^{\pi i / 3}$. By Theorem 0.5 , we see that when $\tau \in \Omega_{5}$ the bifurcation diagram of (0.3) is complicate for $\rho$ ranging from $8 \pi$ to $12 \pi$. It is a natural question to ask if $(0.3)$ has exactly two solutions for $\rho \in(8 \pi, 16 \pi)$ when $\tau \in \Omega_{3}$. Theorem 0.5 also reflects the difficulty in the study the corresponding Lamé equations for the case $n \notin \frac{1}{2} \mathbb{N}$.

\section{ON THE MINIMALITY OF EXTRA CRITICAL POINTS}

Theorem 1.1. Let $p$ be a critical point of $G$ which is not a half period point, then $p$ is a minimal point of $G$. 
Proof. Consider the even, normalized, $L_{2}^{1}$ Sobolev space

$$
H_{e v}^{1}(E)=\left\{u \in H^{1}(E) \mid u(-z)=u(z), \int_{E} u=0\right\}
$$

and the non-linear functional

$$
J_{\rho}(u)=\frac{1}{2} \int_{E}|\nabla u|^{2}-\rho \log \int_{E} e^{-\rho G+u}, \quad u \in H_{e v}^{1}(E) .
$$

It is well known that, as a consequence of the Moser-Trudinger inequality, $J_{\rho}$ attains its minimum for $\rho<8 \pi$. Let $v_{\rho}$ be a minimizer of $J_{\rho}$. Then $v_{\rho}$ is an even solution of

$$
\triangle v+\rho\left(\frac{e^{-\rho G+v}}{\int_{E} e^{-\rho G+v}}-\frac{1}{|E|}\right)=0 \quad \text { in } E .
$$

By the result of [8], when $\rho \rightarrow 8 \pi, v_{\rho}$ converges to a smooth function $v$ which satisfies

$$
\triangle v+8 \pi\left(\frac{e^{-8 \pi G+v}}{\int_{E} e^{-8 \pi G+v}}-\frac{1}{|E|}\right)=0 \quad \text { in } E .
$$

It is then obvious that

$$
u(z)=-8 \pi G(z)+v(z)-\log \int_{E} e^{-8 \pi G+v}
$$

is an even solution to the Liouville equation

$$
\triangle u+8 \pi e^{u}=8 \pi \delta_{0} \quad \text { in } E .
$$

Since

$$
J_{\rho}\left(v_{\rho}\right)=\inf _{\varphi \in H_{e v}^{1}} J_{\rho}(\varphi)
$$

we have

$$
J_{8 \pi}(v)=\inf _{\varphi \in H_{e v}^{1}} J_{8 \pi}(\varphi) .
$$

Let $f$ be the developing map of $u$, that is,

$$
u(z)=\log \frac{8 \pi\left|f^{\prime}(z)\right|^{2}}{\left(1+|f(z)|^{2}\right)^{2}} \quad \text { for } z \in E .
$$

As before, for $\lambda \in \mathbb{R}$ we define $u^{\lambda}$ and $v^{\lambda}$ by

$$
u^{\lambda}(z):=\log \frac{8 \pi e^{2 \lambda}\left|f^{\prime}(z)\right|^{2}}{\left(1+e^{2 \lambda}|f(z)|^{2}\right)^{2}}=: 8 \pi G(z)+v^{\lambda}(z)+c^{\lambda}
$$

where the constant $c^{\lambda}$ is chosen so that $\int_{E} v^{\lambda}=0$. Thus $v^{\lambda}$ is also a solution to (1.2) and $v^{\lambda}(z)$ blows up at $z=p$ as $\lambda \rightarrow+\infty$ (i.e. $p$ is a zero of $f$ ). 
Next we would like to compute $J_{8 \pi}\left(v^{\lambda}\right)$. By differentiation with respect to $\lambda$, we have by (1.2)

$$
\begin{aligned}
\frac{d}{d \lambda} J_{8 \pi}\left(v^{\lambda}\right) & =\int_{E} \nabla v^{\lambda} \cdot \nabla\left(\frac{\partial v^{\lambda}}{\partial \lambda}\right)-8 \pi \frac{\int_{E} e^{-8 \pi G+v^{\lambda}} \frac{\partial v^{\lambda}}{\partial \lambda}}{\int_{E} e^{-8 \pi G+v^{\lambda}}} \\
& =-\int_{E}\left(\triangle v^{\lambda}\right) \frac{\partial v^{\lambda}}{\partial \lambda}-8 \pi \frac{\int_{E} e^{-8 \pi G+v^{\lambda}} \frac{\partial v^{\lambda}}{\partial \lambda}}{\int_{E} e^{-8 \pi G+v^{\lambda}}} \\
& =-\frac{8 \pi}{|E|} \int_{E} \frac{\partial v^{\lambda}}{\partial \lambda}=0 .
\end{aligned}
$$

That is, $J_{8 \pi}\left(v^{\lambda}\right)$ is independent of $\lambda$. In particular,

$$
\lim _{\lambda \rightarrow+\infty} J_{8 \pi}\left(v^{\lambda}\right)=\inf _{\varphi \in H_{e v}^{1}} J_{8 \pi}(\varphi) .
$$

Using (1.4), we shall obtain an upper bound of $\lim J_{8 \pi}\left(v^{\lambda}\right)$ by a choice of suitable test function $\varphi_{\epsilon}$.

We fix a half period point $q \in E$ and small $\delta>0$. For any $\epsilon>0$ we define

$$
\varphi_{\epsilon}(z)= \begin{cases}2 \log \frac{\epsilon^{2} / \delta^{2}+1}{\epsilon^{2}+|z-q|^{2}}+8 \pi \tilde{G}(z, q), & \text { if } z \in B_{\delta}(q), \\ 8 \pi G(z, q), & \text { if } z \in E \backslash B_{\delta}(q),\end{cases}
$$

where

$$
\tilde{G}(z, q)=G(z-q)+\frac{1}{2 \pi} \log |z-q|
$$

is the regular part of $G(z, q)$ which is defined on $z \in T(q)$, the fundamental domain of $E$ centered at $q$. Notice that the above two expressions for $\varphi_{\epsilon}(z)$ coincide when $|z-q|=\delta$. Since $\tilde{G}(z, q)$ depends only on $w=z-q$, we also denote $\tilde{G}(z, q)=\tilde{G}(z-q)=\tilde{G}(w)$, which is defined on the fundamental domain $T(0)$ centered at 0 .

Obviously $\varphi_{\epsilon}$ is an even function. Since $\int_{E} G=0$, direct integration gives

$$
c_{\epsilon}:=\frac{1}{|E|} \int_{E} \varphi_{\epsilon}=\frac{2}{|E|} \int_{B_{\delta}(q)} \log \frac{\left(\epsilon^{2} / \delta^{2}+1\right)|z-q|^{2}}{\epsilon^{2}+|z-q|^{2}}=O\left(\epsilon^{2} \log \epsilon\right)
$$

where the notation $O$ is with respect to the limit $\epsilon \rightarrow 0$. Thus $\varphi_{\epsilon}-c_{\epsilon} \in$ $H_{e v}^{1}(E)$ and

$$
J_{8 \pi}\left(\varphi_{\epsilon}-c_{\epsilon}\right)=\frac{1}{2} \int_{E}\left|\nabla \varphi_{\epsilon}\right|^{2}-8 \pi \log \int_{E} e^{-8 \pi G+\varphi_{\epsilon}}+O\left(\epsilon^{2} \log \epsilon\right) .
$$

We will estimate the energy term and the non-linear term separately. 
By Green's theorem, we have for $w=z-q$,

$$
\begin{aligned}
& \int_{E}\left|\nabla \varphi_{\epsilon}\right|^{2}=\int_{B_{\delta}(q)}\left|\nabla \varphi_{\epsilon}\right|^{2}+(8 \pi)^{2} \int_{E \backslash B_{\delta}(q)}|\nabla G(z-q)|^{2} \\
& =\int_{B_{\delta}(0)} \frac{16|w|^{2}}{\left(\epsilon^{2}+|w|^{2}\right)^{2}} \\
& \quad-32 \pi \int_{B_{\delta}(0)} \log \frac{1}{\epsilon^{2}+|w|^{2}} \triangle \tilde{G}(w)+32 \pi \int_{\partial B_{\delta}(0)} \log \frac{1}{\epsilon^{2}+|w|^{2}} \frac{\partial \tilde{G}(w)}{\partial \nu} \\
& \quad-(8 \pi)^{2} \int_{B_{\delta}(0)} \tilde{G} \triangle \tilde{G}+(8 \pi)^{2} \int_{\partial B_{\delta}(0)} \tilde{G} \frac{\partial \tilde{G}}{\partial \nu} \\
& \quad-(8 \pi)^{2} \int_{E \backslash B_{\delta}(0)} G \triangle G-(8 \pi)^{2} \int_{\partial B_{\delta}(0)} G \frac{\partial G}{\partial \nu} .
\end{aligned}
$$

To estimate these terms, we first notice that (for $\delta>0$ fixed)

$$
\begin{aligned}
\int_{B_{\delta}(0)} \frac{16|w|^{2}}{\left(\epsilon^{2}+|w|^{2}\right)^{2}} & =16 \pi \log \left(1+\delta^{2} / \epsilon^{2}\right)-16 \pi \delta^{2} /\left(\epsilon^{2}+\delta^{2}\right) \\
& =16 \pi\left(\log \left(1+\delta^{2} / \epsilon^{2}\right)-1\right)+O\left(\epsilon^{2}\right) \\
\int_{B_{\delta}(0)} \log \frac{1}{\epsilon^{2}+|w|^{2}} & =O\left(\epsilon^{2} \log \epsilon\right)+O(\delta) .
\end{aligned}
$$

Since $\triangle G=\delta_{0}-1 /|E|, \triangle \tilde{G}=-1 /|E|$, and $\int_{E} G=0$, it is easy to see that each of three integrals involving $G$ or $\tilde{G}$ is $O(\delta)$ and all boundary terms are $O(\delta)$ except

$$
\frac{32 \pi}{\delta} \int_{\partial B_{\delta}(0)} G=32 \pi(-\log \delta+2 \pi \gamma)+O(\delta)
$$

where $\gamma=\tilde{G}(0)=\tilde{G}(q, q)$ is a constant independent of $q$.

Next we compute the non-linear term.

Since both $\nabla G(q)=0$ and $\left.\nabla \tilde{G}(z, q)\right|_{z=q}=\nabla \tilde{G}(0)=0$, we have

$$
\tilde{G}(z, q)-G(z)=\gamma-G(q)+O\left(|z-q|^{2}\right)
$$

and

$$
\begin{aligned}
\int_{B_{\delta}(q)} e^{-8 \pi G(z)+\varphi_{\epsilon}(z)} & =e^{8 \pi(\gamma-G(q))} \int_{B_{\delta}(0)} \frac{\left(\epsilon^{2} / \delta^{2}+1\right)^{2}}{\left(\epsilon^{2}+|w|^{2}\right)^{2}}+O\left(\epsilon^{2} \log \epsilon\right) \\
& =e^{8 \pi(\gamma-G(q))}\left(\frac{\pi}{\epsilon^{2}}-\frac{\pi}{\delta^{2}+\epsilon^{2}}\right)+O\left(\epsilon^{2} \log \epsilon\right) .
\end{aligned}
$$


On $E \backslash B_{\delta}(q)$, by (1.8) and direct estimate we have

$$
\begin{aligned}
\int_{E \backslash B_{\delta}(q)} e^{-8 \pi G+\varphi_{\epsilon}} & =\int_{E \backslash B_{\delta}(q)} e^{8 \pi(G(z, q)-G(z))} \\
& =\int_{T(q) \backslash B_{\delta}(q)} \frac{e^{8 \pi(\tilde{G}(z, q)-G(z))}}{|z-q|^{4}} \\
& =\pi e^{8 \pi(\gamma-G(q))} \frac{1}{\delta^{2}}+O(1),
\end{aligned}
$$

where $O(1)$ denotes a bounded number which is independent of $\delta$ and $\epsilon$.

By taking into account of (1.6)-(1.10), we get for $0<\epsilon<\delta$

$$
\begin{aligned}
J_{8} & \left(\varphi_{\epsilon}-c_{\epsilon}\right) \\
= & 8 \pi\left(\log \left(1+\delta^{2} / \epsilon^{2}\right)-1\right)+16 \pi(-\log \delta+2 \pi \gamma) \\
& -8 \pi \log \pi e^{8 \pi(\gamma-G(q))}\left(\frac{1}{\epsilon^{2}}+\frac{1}{\delta^{2}}-\frac{1}{\epsilon^{2}+\delta^{2}}\right)+O(\delta)+O\left(\epsilon^{2} \log \epsilon\right) \\
= & 64 \pi^{2} G(q)-32 \pi^{2} \gamma-8 \pi(1+\log \pi)+O(\delta)+O\left(\epsilon^{2} \log \epsilon\right) .
\end{aligned}
$$

Let $\epsilon \rightarrow 0$ and then let $\delta \rightarrow 0$. From (1.4) we conclude that

$$
J_{8 \pi}\left(v^{\lambda}\right) \leq 64 \pi^{2} G(q)-32 \pi^{2} \gamma-8 \pi(1+\log \pi) .
$$

From (1.3), $u^{\lambda}$ blows up at $p$ as $\lambda \rightarrow+\infty$. By using the explicit expression (1.3), a similar calculation as the above shows that

$$
\lim _{\lambda \rightarrow+\infty} J_{8 \pi}\left(v^{\lambda}\right)=64 \pi^{2} G(p)-32 \pi^{2} \gamma-8 \pi(1+\log \pi) .
$$

Therefore (1.11) implies

$$
G(p) \leq G(q)
$$

which finishes the proof.

Corollary 1.2. Suppose that $G$ has five critical points. Then any half-period is a saddle point of $G$.

Proof. Since the extra critical point $p$ (reps. $-p$ ) is a discrete minimal point, the index of $\nabla G$ at $p$ (reps. $-p$ ) is 1 . By the Hopf-Poincaré index theorem,

$$
-1=\chi\left(E_{\tau} \backslash\{0\}\right)=2+\sum_{i=1}^{3} \operatorname{ind}_{\frac{1}{2} \omega_{i}} \nabla G .
$$

Since $\frac{1}{2} \omega_{i}$ is non-degenerate, $\nabla G$ has index \pm 1 at it. Hence the index must be -1 for all $i=1,2,3$. This implies that $\frac{1}{2} \omega_{i}$ is a saddle point for all $i$.

\section{COMPUTATIONS FOR $D\left(\frac{1}{2} \omega_{j}\right)$}

Let $u_{k}$ be a sequence of blowup solutions to

$$
\triangle u_{k}+e^{u_{k}}=\rho_{k} \delta_{0}
$$


in $E_{\tau}$ and $\rho_{k} \rightarrow 8 \pi$. Suppose that $\rho_{k} \neq 8 \pi$. In [2, Theorem 0.7.5], it was proved that $u_{k}$ blows up at a half period $q$. Let

$$
\lambda_{k}:=\max _{E_{\tau}} u_{k}
$$

We recall a result in [6]:

Theorem 2.1. Let $\tilde{G}(z, q)$ be the regular part of $G(z, q)$, namely $\tilde{G}(z, q)=G(z-$ $q)+\frac{1}{2 \pi} \log |z-q|$. Let $\phi(q):=\tilde{G}(q, q)$ and $h(z)=e^{-8 \pi G(z)}$. Then

$$
\rho_{k}-8 \pi=(D(q)+o(1)) e^{-\lambda_{k}}
$$

where

$$
D(q):=\int_{E_{\tau}} \frac{h(z) e^{8 \pi(\tilde{G}(z, q)-\phi(q))}-h(q)}{|z-q|^{4}}-\int_{E_{\tau}^{c}} \frac{h(q)}{|z-q|^{4}} .
$$

The quantity $D(q)$ is well defined for any critical point of $G(z, q)$. However, if $q$ is not a half period then $D(q)=0$ since such a blow-up can only occur for $\rho_{k}=8 \pi$. When $q$ is a half period, $D(q)$ has a geometric interpretation. Indeed,

$$
\begin{aligned}
D(q) & =\lim _{r \rightarrow 0}\left(\int_{E_{\tau} \backslash B_{r}(q)} \frac{e^{-8 \pi G(z)} e^{8 \pi(\tilde{G}(z, q)-\phi(q))}}{|z-q|^{4}}-\int_{\mathbb{R}^{2} \backslash B_{r}(q)} \frac{e^{-8 \pi G(q)}}{|z-q|^{4}}\right) \\
& =\lim _{r \rightarrow 0}\left(\int_{E_{\tau} \backslash B_{r}(q)} e^{-8 \pi \phi(q)} e^{8 \pi(G(z-q)-G(z))}-\int_{\mathbb{R}^{2} \backslash B_{r}(q)} \frac{e^{-8 \pi G(q)}}{|z-q|^{4}}\right) .
\end{aligned}
$$

Note that $8 \pi(G(z-q)-G(z))$ is a doubly periodic harmonic function in $\mathbb{R}^{2}$ with singularities $-4 \log |z-q|$ at $z=q$ and $4 \log |z|$ at $z=0$. Thus

$$
8 \pi(G(z-q)-G(z))=2 \log |\wp(z-q)-\wp(q)|+C
$$

for the constant $C=8 \pi(\phi(q)-G(q))$. (The identity does not hold if $q$ is not a half period.) Therefore,

$$
e^{8 \pi(G(z-q)-G(z))}=e^{8 \pi(\phi(q)-G(q))}|\wp(z-q)-\wp(q)|^{2},
$$

and

$$
D(q)=e^{-8 \pi G(q)} \lim _{r \rightarrow 0}\left(\int_{E_{\tau} \backslash B_{r}(0)}|\wp(z)-\wp(q)|^{2}-\int_{\mathbb{R}^{2} \backslash B_{r}(0)} \frac{1}{|z|^{4}}\right) .
$$

Let $T$ be a fundamental domain of $E_{\tau}$ with $0 \notin \partial T$. Let $\gamma$ be the image of $\Gamma:=\partial T$ under the map

$$
\Sigma(z):=-\zeta(z)-\wp(q) z
$$

Denote by $\Lambda_{+}(q)$ be the union of components bounded by $\gamma$ and covered by $T$ under $\Sigma$, and by $\Lambda_{-}(q)$ the union of components bounded by $\gamma$ but not covered by $T$ under $\Sigma$. Then obviously

$$
\left|\Lambda_{+}(q)\right|-\left|\Lambda_{-}(q)\right|=\lim _{r \rightarrow 0}\left(\int_{E_{\tau} \backslash B_{r}(0)}|\wp(z)-\wp(q)|^{2}-\int_{\mathbb{R}^{2} \backslash B_{r}(0)} \frac{1}{|z|^{4}}\right)
$$


and so

$$
D(q)=e^{-8 \pi G(q)}\left(\left|\Lambda_{+}(q)\right|-\left|\Lambda_{-}(q)\right|\right) .
$$

We will give another characterization of $D(q)$ in terms of the Hessian of $G$ at $q$, hence establish a correspondence between the geometric interpretation and the degeneracy structure of the Green function. Recall [8, (7.7)]:

$$
\operatorname{det} D^{2} G=\frac{-1}{4 \pi^{2}}\left(\left|(\log \vartheta)_{z z}\right|^{2}+\frac{2 \pi}{b} \operatorname{Re}(\log \vartheta)_{z z}\right) .
$$

To write it in the Weierstrass theory we use $(\log \vartheta)_{z}(z)=\zeta(z)-\eta_{1} z$ and

$$
(\log \vartheta)_{z z}\left(\frac{1}{2} \omega_{i}\right)=-\wp\left(\frac{1}{2} \omega_{i}\right)-\eta_{1}=-\left(e_{i}+\eta_{1}\right) .
$$

Theorem 2.2. For any half period $q$,

$$
\left|\Lambda_{+}(q)\right|-\left|\Lambda_{-}(q)\right|=-4 \pi^{2} b \operatorname{det} D^{2} G(q) .
$$

Proof. Without loss of generality, we assume that $q=\frac{1}{2} \omega_{1}=\frac{1}{2}$ and denote $\Lambda_{+}(q)$ and $\Lambda_{-}(q)$ by $\Lambda_{+}$and $\Lambda_{-}$respectively. By (2.2), we have

$$
\begin{aligned}
\left|\Lambda_{+}\right|-\left|\Lambda_{-}\right|= & \lim _{r \rightarrow 0}\left(\int_{E_{\tau} \backslash B_{r}(0)}|\wp(z)|^{2}-\int_{\mathbb{R}^{2} \backslash B_{r}(0)} \frac{1}{|z|^{4}}\right) \\
& -\lim _{r \rightarrow 0} \int_{E_{\tau} \backslash B_{r}(0)}\left(\wp(z) \bar{e}_{1}+\bar{\wp}(z) e_{1}\right)+b\left|e_{1}\right|^{2},
\end{aligned}
$$

where $\tau=a+b i$.

To compute the first term, write the Weierstrass zeta function as $\zeta=$ $u+i v$ and then $\wp=-\zeta^{\prime}=-u_{x}-i v_{x}=-u_{x}+i u_{y}$. Hence

$$
|\wp|^{2}=u_{x}^{2}+u_{y}^{2}=\partial_{x}\left(u u_{x}\right)+\partial_{y}\left(u u_{y}\right) .
$$

Using integration by parts, and noticing that the singularity at $z=0$ is cancelled out by the second integral, the first limit term then becomes

$$
\int_{\Gamma} u u_{x} d y-u u_{y} d x=\int_{\Gamma} u\left(v_{x} d x+v_{y} d y\right)=\int_{\Gamma} u d v
$$

This can be calculated easily as

$$
-\frac{1}{2} \operatorname{Im} \int_{\Gamma} \zeta d \bar{\zeta}=\frac{1}{2} \operatorname{Im}\left(\bar{\eta}_{1} \eta_{2}-\eta_{1} \bar{\eta}_{2}\right)
$$

Applying the Legendre relation $\eta_{2}=\eta_{1} \tau-2 \pi i$, we get

$$
\bar{\eta}_{1} \eta_{2}-\eta_{1} \bar{\eta}_{2}=\bar{\eta}_{1}\left(\eta_{1} \tau-2 \pi i\right)-\eta_{1}\left(\bar{\eta}_{1} \bar{\tau}+2 \pi i\right)=2 i b\left|\eta_{1}\right|^{2}-2 \pi i\left(\eta_{1}+\bar{\eta}_{1}\right) .
$$

Consequently,

$$
\lim _{r \rightarrow 0}\left(\int_{E_{\tau} \backslash B_{r}(0)}|\wp(z)|^{2}-\int_{\mathbb{R}^{2} \backslash B_{r}(0)} \frac{1}{|z|^{4}}\right)=b\left|\eta_{1}\right|^{2}-\pi\left(\eta_{1}+\bar{\eta}_{1}\right) .
$$


For the second limit term, we first compute

$$
\begin{aligned}
\int_{E_{\tau} \backslash B_{r}(0)} \wp(z) & =\frac{i}{2} \int_{T \backslash B_{r}(0)} \wp d z \wedge d \bar{z}=-\frac{i}{2} \int_{T \backslash B_{r}(0)} d(\zeta d \bar{z}) \\
& =-\frac{i}{2}\left(\int_{\Gamma} \zeta d \bar{z}-\int_{\partial B_{r}(0)} \zeta d \bar{z}\right) .
\end{aligned}
$$

This first integral gives $\eta_{1} \bar{\tau}-\eta_{2}=\eta_{1} \bar{\tau}-\eta_{1} \tau+2 \pi i=-2 b i \eta_{1}+2 \pi i$. For the second integral, in the limit $r \rightarrow 0$ it tends to $\int_{0}^{2 \pi} e^{-i \theta} e^{-i \theta}(-i) d \theta=0$. Hence

$$
\lim _{r \rightarrow 0} \int_{E_{\tau} \backslash B_{r}(0)} \wp(z)=-\eta_{1} b+\pi .
$$

Putting everything together we get (c.f. (2.4) and (2.5))

$$
\begin{aligned}
& \left|\Lambda_{+}\right|-\left|\Lambda_{-}\right| \\
& =b\left|\eta_{1}\right|^{2}-\pi\left(\eta_{1}+\bar{\eta}_{1}\right)+\left(\eta_{1} b-\pi\right) \bar{e}_{1}+\left(\bar{\eta}_{1} b-\pi\right) e_{1}+b\left|e_{1}\right|^{2} \\
& =b\left|e_{1}+\eta_{1}\right|^{2}-\pi\left(\left(e_{1}+\eta_{1}\right)+\left(\overline{e_{1}+\eta_{1}}\right)\right) \\
& =-4 \pi^{2} b \operatorname{det} D^{2} G\left(\frac{1}{2} ; \tau\right) .
\end{aligned}
$$

The proof is completed.

Corollary 2.3. Let $u_{k}$ be a sequence of blow-up solutions to (2.1) with $\rho_{k} \rightarrow 8 \pi$ and $q$ the blow-up point.

(1) $q$ is a half period and a saddle point of $G(z ; \tau)$ if and only if $\rho_{k}>8 \pi$.

(2) $q$ is a half period and a minimal point of $G(z ; \tau)$ if and only if $\rho_{k}<8 \pi$.

\section{UNIQUENESS OF SOLUTIONS}

In this section we classify all solutions to

$$
\triangle u+e^{u}=\rho \delta_{0} \quad \text { on } E
$$

for $0<\rho \leq 8 \pi+\epsilon_{0}$ where $\epsilon_{0}$ is a small positive number.

Recall in [8] we showed that equation (3.1) has a unique solution for $\rho=4 \pi$, and a unique even solution for $4 \pi \leq \rho \leq 8 \pi$. Here we prove the uniqueness result without the evenness assumption.

Lemma 3.1. Equation (3.1) has a unique solution for $0<\rho \leq 4 \pi$.

Proof. We first show that for any solution $u$ to (3.1) with $\rho \leq 4 \pi$, the linearized equation

$$
\triangle \phi+e^{u} \phi=0 \quad \text { on } E
$$

has only trivial solution $\phi=0$.

Suppose that $\phi$ is a solution to (3.2). Then a straightforward computation shows that $\left(\phi_{z z}-u_{z} \phi_{z}\right)_{\bar{z}}=0$. Since

$$
u(z) \sim \frac{\rho}{2 \pi} \log |z|,
$$


$\phi_{z z}-u_{z} \phi_{z}$ is an elliptic function on $E$ whose only singularity is a pole of order one at 0 . This forces that $\phi_{z}(0)=0$ and

$$
\phi_{z z}-u_{z} \phi_{z}=c_{1} \quad \text { on } E
$$

for some constant $c_{1}$, or equivalenly

$$
\left(e^{-u} \phi_{z}\right)_{z}=c_{1} e^{-u}
$$

Notice that

$$
\left|e^{-u} \phi_{z}(z)\right| \leq c_{2}|z|^{1-\rho / 2 \pi}
$$

for some constant $c_{2}>0$. Thus if $\rho<4 \pi$,

$$
\lim _{r \rightarrow 0} \int_{E \backslash B_{r}(0)}\left(e^{-u} \phi_{z}\right)_{z}=\frac{1}{2} \lim _{r \rightarrow 0} \int_{\partial B_{r}(0)} e^{-u} \phi_{z} \frac{\bar{z}}{|z|} d s=0,
$$

and if $\rho=4 \pi$ the above limit is finite. If $c_{1} \neq 0$, this implies that

$$
\int_{E} e^{-u}= \begin{cases}0 & \text { if } \rho<4 \pi \\ <\infty & \text { if } \rho=4 \pi\end{cases}
$$

which leads to a contradiction. So we have $c_{1}=0$ and $e^{-u} \phi_{\bar{z}}$ is an elliptic function. By (3.3) this again implies that $e^{-u} \phi_{\bar{z}}=c_{3}$ is a constant.

If $\phi \not \equiv 0$ then $\phi$ has a maximum point $p$ and a minimum point $q$ with $p \neq q$. One of $p, q$ is not a lattice point where $\phi_{\bar{z}}=0$. This implies that $c_{3}=0$ and hence $\phi_{\bar{z}} \equiv 0$. This leads to $\phi \equiv 0$ which is a contradiction to $\phi \not \equiv 0$. Hence we must have $\phi \equiv 0$.

Now the uniqueness follows from the fact that (3.1) has only one solution at $\rho=4 \pi$.

Remark 3.2. In [8] we showed that the unique even solution to (3.1) with $\rho \in$ $[4 \pi, 8 \pi]$ is non-degenerate in the class of $H_{e v}^{1}=\left\{u \in H^{1} \mid u(-z)=u(z)\right\}$. Now the proof of Lemma 3.1 allows us to remove the evenness assumption: $u$ is non-degenerate in the whole space $H^{1}$, provided that $0<\rho<8 \pi$.

To see this, we may assume that the solution $\phi$ is odd. Therefore $\phi_{z z}-$ $u_{z} \phi_{z}$ is odd and by exactly the same calculation we have

$$
\phi_{z z}-u_{z} \phi_{z}=c_{1}=0 \text {. }
$$

This implies that $e^{-u} \phi_{\bar{z}}$ is an elliptic function on $E$, with 0 being its only pole. However, since $\phi$ is odd, $\phi_{\bar{z}}$ is even and the estimate (3.3) can be improved to

$$
\left|e^{-u} \phi_{z}(z)\right| \leq c_{2}|z|^{2-\rho / 2 \pi}
$$

If $\rho<8 \pi$, we find $2-\rho / 2 \pi>-2$. This implies that $e^{-u} \phi_{\bar{z}}$ is a constant. If $\phi \not \equiv 0$, by evaluating it at a maximum or minimum point, with one of it not a lattice point, we conclude that $e^{-u} \phi_{\bar{z}} \equiv 0$, and then $\phi \equiv 0$ follows. (Notice that if $\rho=8 \pi$ then $e^{-u} \phi_{\bar{z}}=c \wp(z)$ for some constant $c \neq 0$.)

Now we may conclude that the unique even solution $u$ is always a minimum point of the non-linear functional $J_{\rho}$ in (1.1) for $0<\rho \leq 8 \pi$. In fact we can prove a stronger result, namely Theorem 0.5 . 
Lemma 3.3. Let $u$ be a solution to (3.1) with $\rho \notin 8 \pi \mathbb{N}$. Then $u$ is even.

Proof. This was proved in [2] for $\rho=4 \pi l$ with $l$ being a positive odd integer, so we assume that $\rho \notin 4 \pi \mathbb{N}$.

Let $f(z)$ be a multi-valued developing map of $u$. The readers are referred to $[2, \S 8]$ for the details to treat these multi-valued functions as global analytic functions $\mathbf{f}(\xi)$, which are defined on the universal cover $\xi \in \mathbb{H} \rightarrow E^{\times}$. In particular the cusp $\xi=0$ is mapped to the cusp $z=0$ in $E^{\times}$.

As in [2], we have $S(f)=2(\eta(\eta+1) \wp+B)$ for $\eta=\rho / 8 \pi$ for some $B \in \mathbb{C}$. Thus $f=w_{1} / w_{2}$ and $\mathbf{f}=\mathbf{w}_{1} / \mathbf{w}_{2}$ for two linearly independent solutions $w_{1}$ and $w_{2}$ to the Lamé equation

$$
w^{\prime \prime}=(\eta(\eta+1) \wp+B) w .
$$

Since $\tilde{w}_{i}(z):=w_{i}(-z)$ are also two linearly independent solutions to (3.4), $\tilde{f}:=\tilde{w}_{1} / \tilde{w}_{2}$ also defines a global analytic function $\tilde{\mathbf{f}}$ and we have

$$
\tilde{\mathbf{f}}=S \mathbf{f}=\frac{a \mathbf{f}+b}{c \mathbf{f}+d^{\prime}}, \quad \text { for some } \quad S=\left(\begin{array}{ll}
a & b \\
c & d
\end{array}\right) \in \operatorname{SL}(2, \mathbb{C}) .
$$

Consider the covering transformations on $\mathbb{H}: g_{1}, g_{2} \in \mathrm{SL}(2, \mathbb{R})$ determined by the two free generators of $\pi_{1}\left(E^{\times}\right) \cong \mathbb{Z} * \mathbb{Z}$. Let $\Gamma<\operatorname{SL}(2, \mathbb{R})$ be the rank two free subgroup generated by $g_{1}$ and $g_{2}$, and $r: \Gamma \rightarrow \operatorname{PSU}(2)$ be the unitary representation associated to the solution $u$. The mapping $(-1): z \mapsto-z$ on $E^{\times}$lifts to a map $\iota$ on $\mathbb{H}$ which is not a covering map for $\mathbb{H} \rightarrow E^{\times}$. Nevertheless the composition $\iota \circ \iota$, namely we apply $(-1)$ twice, does give a covering map for $\mathbb{H} \rightarrow E^{\times}$. That is, the matrix $S^{2}$ can be represented as an element generated by $S_{1}:=r\left(g_{1}\right)$ and $S_{2}:=r\left(g_{2}\right)$.

By considering the action of $(-1)$ in a simply connected neighborhood $U$ of $0 \in E$, we see that $S^{2} f=f\left(e^{2 \pi i} z\right)=\beta f(z)$ for some $\beta \in \operatorname{PSU}(2, \mathbb{C})$. Indeed, $\beta=r\left(g_{2}^{-1} g_{1}^{-1} g_{2} g_{1}\right)=S_{2}^{-1} S_{1}^{-1} S_{2} S_{1}$. Under some normalization on $\mathrm{f}$, the matrix $\beta$ is calculated in [2, Lemma 8.3.4, p.262] (suppress all index $k$ in the formula in the bottom of p.262) as

$$
\beta=\left(\begin{array}{cc}
|p|^{2} \alpha+|q|^{2} \bar{\alpha} & -\bar{p} q(\alpha-\bar{\alpha}) \\
p \bar{q}(\bar{\alpha}-\alpha) & |p|^{2} \bar{\alpha}+|q|^{2} \alpha
\end{array}\right)
$$

where $\mathbf{f}(0):=\lim _{\tilde{\xi} \rightarrow 0} \mathbf{f}(\xi)=q / p$ with $|p|^{2}+|q|^{2}=1, p, q \neq 0$, and $\alpha=$ $e^{2 \pi i \eta}$. Clearly $\alpha \neq \bar{\alpha}$ since $\eta \notin \frac{1}{2} \mathbb{Z}$. In particular $\beta \neq \pm I_{2}$.

We claim that $S \in \operatorname{PSU}(2, \mathbb{C})$. To prove it, we choose a new unitary basis to diagonalize $\beta$ to $\left(\begin{array}{cc}e^{i \theta} & 0 \\ 0 & e^{-i \theta}\end{array}\right)$ for some $e^{i \theta} \neq \pm 1$. Since

$$
S^{2}=\left(\begin{array}{cc}
a^{2}+b c & b(a+d) \\
c(a+d) & d^{2}+b c
\end{array}\right),
$$

$S^{2}=\beta$ implies that either $a+d=0$ or both $b=0$ and $c=0$. If $a+d=0$ then $a^{2}+b c=-a d+b c=-1$ and $d^{2}+b c=-1$, which leads to $e^{i \theta}=-1$, a contradiction. Hence $b=c=0$ and $a d=1, a^{2}=e^{i \theta}, d^{2}=e^{-i \theta}$. Therefore, 
$S \in \operatorname{PSU}(2, \mathbb{C})$ and $\tilde{f}(z)=f(-z)$ gives rise to the same solution $u$. Hence $u(-z)=u(z)$ and the the lemma follows.

Proof of Theorem 0.5. By Lemma 3.1 and 3.3, the uniqueness of solution holds for $0<\rho<8 \pi$. The statements for $\rho=8 \pi$ was proved in [8].

For $\tau \in \Omega_{3}$, the unique solutions $u_{\rho}$ blows up as $\rho \nearrow 8 \pi$ (since equation (3.1) has no solutions at $\rho=8 \pi$ ). The blow-up point of $u_{\rho}$ must be the minimum point which is one of the half periods. The other two half periods $q_{1}$ and $q_{2}$ are saddle critical point of $G$. By Theorem 0.4 and Remark 0.3 , we have det $D^{2} G\left(q_{i}\right)<0$ and then $D\left(q_{i}\right)>0$. Under these conditions, by the method in [4] we can construct a bubbling sequence of solutions $u_{\rho, i}$ to (3.1), for each $i=1,2$, with $\rho>8 \pi$ which blows up at $q_{i}$.

Remark 3.4. In [4] the non-degenerate condition $D\left(q_{i}\right) \neq 0$ was replaced by some other non-degenerate condition. Nevertheless the similar process as there still works in our current case (see e.g. the remark in [6] concerning with the degree counting formula).

Indeed, for the Chern-Simons-Higgs equation, the same non-degenerate conditions $D(q)<0$ and $\operatorname{det} D^{2} G(q) \neq 0$ were recently used to construct such kind of bubbling solutions [10].

Now we need the following uniqueness theorem:

Theorem 3.5. Suppose that $u_{k}$ and $\tilde{u}_{k}$ are two sequences of solutions to (3.1) with $\rho_{k} \rightarrow 8 \pi$, and both sequences have the same blow-up point $q$.

If $D(q) \neq 0$, i.e. $q$ is a non-degenerate critical point of $G$ by Theorem 0.4 , then $u_{k}=\tilde{u}_{k}$ for large $k$.

This is recently proved in [11] for the Chern-Simons-Higgs equation

$$
\triangle u+\frac{1}{\epsilon} e^{u}\left(1-e^{u}\right)=8 \pi \delta_{0},
$$

but the proof given there also works for (3.1).

By Theorem 3.5, $u_{\rho, i}$ are exactly all the solutions to equation (3.1) for $8 \pi<\rho<8 \pi+\epsilon_{0}$. This proves (i).

For $\tau \in \Omega_{5}$, all the three half periods are saddle points of $G$. By Theorem 3.5 again, we must have three bubbling solutions. On the other hand, (3.1) has a unique even solution $u$ for $\rho=8 \pi$ whose linearized equation in the class of even functions is non-degenerate. Therefore for $8 \pi<\rho<8 \pi+\epsilon_{0}$ there is a unique even solution $u_{\rho}$ which converges to to $u$ as $\rho \searrow 8 \pi$.

By Lemma 3.3, (3.1) has only even solutions for $8 \pi<\rho<8 \pi+\epsilon_{0}$, we conclude that (3.1) has the only one even solution $u_{\rho}$ which converges to $u$ as $\rho \searrow 8 \pi$. Hence there are four solutions in total. This proves (ii) and thus completes the proof Theorem 0.5.

\section{REFERENCES}

[1] S.-Y. Chang, C.-C. Chen and C.-S. Lin; Extremal functions for a mean field equation in two dimension, Lectures on PDE, 61-93, New Stud. Adv. Math. 2, Int. Press, 2003. 
[2] C.-L. Chai, C.-S. Lin and C.-L. Wang; Mean field equations, hyperelliptic curves, and modular forms: I, Cambridge J. of Math. 3 (2015), no. 1-2, 127-274.

[3] C.-C. Chen and C.-S. Lin; Sharp estimates for solutions of multi-bubbles in compact Riemann surfaces, Comm. Pure Appl. Math. 55 (2002), 728-771.

[4] — Topological degree for a mean field equation on a Riemann surface, Comm. Pure Appl. Math. 56 (2003), 1667-1727.

[5] — Topological degree for a mean field equation with singular sources, Comm. Pure Appl. Math. 68 (2015), 887-947.

[6] C.-C. Chen, C.-S. Lin and G. Wang; Concentration phenomenon of two-vortex solutions in a Chern-Simons model, Ann. Scuola Norm. Sup. Pisa CI. Sci. (5) Vol. III (2004), 367-379.

[7] Z. Chen, K.-J. Kuo, C.-S. Lin and C.-L. Wang; Green function, Painlevé VI equation, and Eisentein series of weight one, preprint 2015.

[8] C.-S. Lin and C.-L. Wang; Elliptic functions, Green functions and the mean field equations on tori, Annals of Math. 172 (2010), no.2, 911-954.

[9] — ; A function theoretic view of the mean field equations on tori, in "Recent advances in geometric analysis", 173-193, Adv. Lect. Math. 11, Int. Press, Somerville MA, 2010.

[10] C.-S. Lin and S.-S. Yan; Existence of bubbling solutions for Chern-Simons model on a torus, Arch. Ration. Mech. Anal. 207 (2013), no. 2, 352-392.

[11] _ - On the self-dual condensate of the Chern-Simons-Higgs model, Part II: local uniqueness and applications, preprint 2014.

[12] M. Nolasco and G. Tarantello; Double vortex condensation in the Chern-Simons-Higgs theory, Calc. Var. Partial Diff. Equ. 9 (1999), 31-94.

Department of MATHEMATICS AND CENTER FOR AdVANCED STUdies IN THEORETIC SCIENCES (CASTS), NATIONAL TAIWAN UNIVERSITY, TAIPEI

E-mail address: cslinemath.ntu.edu.tw

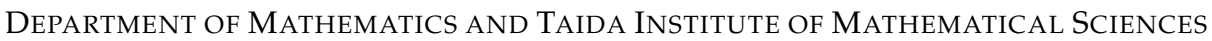
(TIMS), NATIONAL TAIWAN UNIVERSITY, TAIPEI

E-mail address: dragon@math.ntu.edu.tw 